\title{
STRUCTURE-BASED DRUG DESIGNING STUDIES TOWARDS EXPLORING THE POTENTIAL ANTICANCER ACTIVITY OF SELECTED PHYTOCOMPOUNDS AGAINST HISTONE DEACETYLASE 10 PROTEIN
}

\author{
SABEENA M ${ }^{1,2}$, KAISER JAMIL ${ }^{3 *}$, SWAMY AVN $^{4}$ \\ ${ }^{1}$ Centre for Biotechnology and Bioinformatics, Jawaharlal Nehru Institute of Advanced Studies, Buddha Bhavan, Secunderabad, Telangana, \\ India. ${ }^{2}$ Department of Biotechnology, Jawaharlal Nehru Technological University Anantapur, Ananthapuramu, Andhra Pradesh, India. \\ ${ }^{3}$ Department of Genetics, Bhagwan Mahavir Medical Research Centre 10-1-1, Mahavir Marg, AC Guards, Hyderabad, Telangana, India. \\ ${ }^{4}$ Department of Chemical Engineering, Jawaharlal Nehru Technological University Anantapur, Ananthapuramu, Andhra Pradesh, India. \\ Email: kj.bmmrc@gmail.com
}

Received: 14 June 2018, Revised and Accepted: 01 August 2018

ABSTRACT

Objective: Histone deacetylases (HDACs) are proteins which play a crucial role in cell growth, maintenance, and regulation. Abnormal HDAC proteins produced by genetic mutations are common in human cancers. HDAC10 is a class II HDAC member, and its expression in many cancers has been documented. The aim of this study was to determine the best docking of phytocompounds selected from a list of such compounds in the database of chemicals for HDAC10.

Methods: The crystal structure of HDAC10 was retrieved from Protein Data Bank and prepared for docking studies by post-translational modification (PTM) analysis. Then, we have screened 450 phytocompounds for molecular docking studies and determined their binding affinities against HDAC10 by using PatchDock server.

Results: The PTM analysis showed that myristoylation sites were more abundant in HDAC10 which might be important functional sites for the gene regulation. The results revealed the receptor/inhibitor interactions within an active domain consisting of 30 important amino acid residues. Affinitybased studies have indicated the docking energy levels by calculating hydrogen bonding, steric, and hydrophobic interactions. Among the inhibitors, we could shortlist four compounds which showed excellent binding affinity. Hence, we evaluated drug binding affinities of these four compounds and determined their atomic contact energy values. Analysis of the docking results showed holacurtine>periplogenin>3,3'-diindolylmethane>epigallocat echin as the order of binding affinities, with holacurtine having the best docking score.

Conclusion: It is proposed from these studies that the docking and scoring methods could be useful for selecting and shortlisting the promising antitumor molecules. These molecules could be further tested using in vitro and in vivo methods to confirm their role in HDAC10-associated cancers. Furthermore, myristoylation sites in HDAC10 could form an important binding site for selecting hit inhibitor compounds. The PTM studies together with the binding mode analysis facilitate the protein-protein interaction studies of HDAC10, and thioredoxin-interacting protein is considered as one of the transcriptional regulators of HDAC10.

Keywords: Histone deacetylase 10, Post-translational modifications, Phytocompounds, Docking studies, Thioredoxin-interacting protein.

(C) 2018 The Authors. Published by Innovare Academic Sciences Pvt Ltd. This is an open access article under the CC BY license (http://creativecommons. org/licenses/by/4. 0/) DOI: http://dx.doi.org/10.22159/ajpcr.2018.v11i12.27944

\section{INTRODUCTION}

Histones are the basic proteins, and its acetylation process is a key prerequisite for chromatin decondensation and structure [1]. Studies confirm that histones play a pivotal role in the regulation of chromatin structure, function, and dynamics [2,3]. Researches provide the information regarding known histone acetyltransferases (HAT) and histone deacetylases (HDACs) and their effects on transcription of target genes in vivo [4]. HDACs regulate various cellular processes through enzymatic deacetylation of both histone and non-histone protein [5]. These proteins play crucial roles in a variety of biological processes including cell cycle progression, proliferation, differentiation, and development [6]. The role of HDAC in cancer was studied for the first time in 1998 [7]. Several recent studies also have implicated that individual HDAC enzymes can form potential therapeutic targets for treatment against cancers, fibrotic disorders, immune and neurological disorders [8-10]. HDAC family consists of two major classes, namely class I and class II, based on the homologies to yeast deacetylases both share a highly conserved catalytic domain which is considered as receptor for many drugs, especially in cancer studies [11]. Hence, HDAC inhibitors (HDACi) represent a new class of targeted anticancer agents [12].
HDACi has extensively demonstrated the antitumor efficacy in vitro and in vivo. In vitro experiments in cancer cell cultures and in vivo studies using mouse xenograft model have shown that HDACi delivers potent anticancer effects [13]. Therefore, the determination of HDACi has become one of the most important research fields of the anticancer drugs. Several classes of HDACi have been found to have potential anticancer activities and undergoing preclinical studies [14]. Studies have reported that HDAC class II proteins were modeled and two known inhibitors, namely suberoylanilide hydroxamic acid (SAHA) and trichostatin A, were docked using AutoDock to identify the pharmacological properties [15]. The inhibition of HDAC activity leads to the modulation of expression of a specific set of genes which can result in growth arrest, differentiation and apoptosis [16].

HDAC10 is a member of class II HDAC which was first discovered based on sequence homology to other class II HDACs and is widely expressed in adult human tissues and cultured mammalian cells. It is a 669 residue polypeptide with a bipartite modular structure consisting of an $\mathrm{N}$-terminal Hda1p-related putative deacetylase domain and a C-terminal leucine-rich domain [17]. Its mutation leads to various cancers [18-20]. Homology comparison indicates that HDAC10 is most 
similar to HDAC6. Both contain a unique, putative catalytic domain which is not found in other HDACs. In HDAC10, however, this domain is not functional [21]. HDAC6 is a cytoplasmic deacetylase while HDAC10 is known to occur in both the nucleus and the cytoplasm. In the nucleus, HDAC10 contains a distinct transcriptional repressor domain. It is found that HDAC10 might uniquely play roles both in the nucleus, as a transcriptional modulator, and in the cytoplasm in an unidentified role [21]. It has been found that the knockdown of HDAC10 significantly increased the mRNA expression levels of thioredoxin-interacting protein (TXNIP) in human gastric cancer cells [22]. Real-time PCR and immunoblot analysis confirmed that inhibition of HDAC10 induced TXNIP expression, more specifically which is involved in transcriptional downregulation of TXNIP. In our previous study, we identified the drug binding mechanism of TXNIP [23]. The expression levels of HDAC10 decrease in lung cancer patients class II HDACs repress critical genes and eventually cause the progression of lung cancer [24]. It has been found that HDAC10 acts as a promoter of autophagy-mediated survival in neuroblastoma cells [25] and chronic lymphocytic leukemia [26].

Only two drug compounds, namely belinostat [27] and panobinostat [28], are currently approved as inhibitors for HDAC10. This leads to increasing demands for additional therapeutics options by administering natural and phytocompounds with lesser side effects. Thus, our study is an attempt to determine the binding efficacy of a series of phytocompounds and evaluate their interaction with HDAC10. To achieve this goal, first, we have used computational algorithms to determine the different post-translational modification sites (PTMs) and binding sites. Then, docking and its simulation studies were performed. We have used several structural algorithms to identify binding sites, through analyzing surface structures. In addition, we focus mainly on the selection of compounds, and we evaluated the binding mode of selected phytocompounds for docking and scoring studies. Further, in this study, we also able to understand the drug docking mechanism of HDAC10 whether it can give a clue for noncoding regions and identify conserved sequence elements that may be involved in its gene regulation process.

\section{METHODS}

\section{HDAC-10 sequence retrieval and its PTMs studies}

The query sequence of HDAC10 was retrieved from The UniProt Knowledgebase (UniProtKBid: Q969S8). UniProtKB acts as a central hub of protein knowledge by providing a unified view of protein sequence and functional information [29]. To predict functional sites of HDAC10, the query sequence was submitted to Expasy ScanProsite online server (https://prosite.expasy.org/scanprosite/) which is a database used for structurally conserved motifs scanning included UniProtKB/SwissProt, splice variants, and UniProtKB/TrEMBL databases. All the default settings were considered as parameters for the analysis.

\section{HDAC10 receptor preparation}

The receptor structure of HDAC10 was prepared by retrieving the three-dimensional crystal structure complex (Protein Data Bank [PDB] id:5TD7) bound with a ligand structure, namely 7-[(3-aminopropyl) amino]-1,1,1-trifluoroheptane-2,2-diol, from RCSB PDB (http://www. rcsb.org/pdb/home/home.do) [30]. The protein was cleaned by removing the bound molecule and the non-essential water molecules. Hydrogen atoms were added to the receptor using Biovia DS visualizer (Accelrys, BioVia, San Diego, CA, USA).

\section{Binding pocket recognition}

The probable ligand binding pockets in HDAC10 were predicted and visually identified by MetaPocket server (http://projects.biotec.tudresden.de/metapocket/), which is an online tool that locates and measures pockets in the protein structures [31].

\section{Molecules retrieval and ligand preparation}

The chemical structures of 450 phytocompounds were known to have anticancer activities which were downloaded from NCBI PubChem (https://pubchem.ncbi.nlm.nih.gov/) in Standard Data Format (SDF).
PubChem is an open repository for chemical structures and their biological test results [32]. The SDF files were converted to 3D structures such as mol2 and PDB using Open Babel [33] converter and visualized using Biovia DS visualizer. These molecules were further taken for molecular docking studies.

\section{Docking studies using the selected phytocompounds}

A geometry-based molecular docking algorithm, namely PatchDock server (http://bioinfo3d.cs.tau.ac.il/PatchDock) [34,35], was used for docking of HDAC10 with the selected phytocompounds. PatchDock is an online docking server which is designed for the purpose of the identification of the interaction sites between the receptor and ligand molecules. The molecular surface of the protein is divided into patches as per the molecular shape followed by the comparison between the patches to produce a group of transformations [36]. These transformations were further ranked based on the geometric complementarity score, and each transformation was assigned to have a score, namely PatchDock score and atomic contact energy (ACE) values.

\section{Analysis of docking results}

To optimize the observed anti-inflammatory and anticancer activities from literature studies, we evaluated the binding mode of each docked complexes. In this step, the generated poses of each docked complex were analyzed and ranked based on their predicted binding energies, termed as ACE value. These rankings were used to evaluate the binding affinity of the receptor to develop the best conformational fit with the ligands selected for analysis. Among the generated multiple conformations and associated binding energies, the pose with least energy values was considered as the best pose and reported using Biovia DS visualizer. The atomic dissolution energy of the derive complex was estimated, and the redundant solution was calculated using RMSD, clustering during the docking process [34,37]. The docking poses with interacting amino acids were graphically represented by Biovia DS 4.5. The best poses were analyzed for hydrogen bonding, steric, and hydrophobic interactions.

Drug-likeness and Lipinski's rule of five calculation and pharmacokinetics evaluation

Molecular descriptors and drug likeliness properties of the selected phytocompounds were evaluated using the online tool named as Molinspiration server (http://www.molinspiration.com), based on Lipinski Rules of five [38]. The smiles format of each of the compound was uploaded for the analysis. As per Lipinski's rule, "drug-like" molecules must have $\log p \leq 5$, molecular weight $\leq 500$, number of hydrogen bond acceptors $\leq 10$, and number of hydrogen bond donors $\leq 5$.

\section{RESULTS AND DISCUSSION}

\section{PTM analysis of HDAC10}

According to prediction results, there were 25 hits for PTMs which resulted as three patterns. Protein kinase C phosphorylation, Casein kinase II phosphorylation, and $\mathrm{N}$-myristoylation sites were predicted as critical biochemical events required for HDAC10 regulation. Three regions were identified as protein kinase $C$ phosphorylation sites (amino acid residues range from 51 to 53,345 to 347 , and 409 to 411). The residues occurring at 417-420 were identified as phosphothreonine, T, whereas others were phosphoserine, S. Protein kinase C appeared to react with serine or threonine residues that were located at the aminoterminal (C terminal) side close to lysine or arginine [39]. The results showed the low level score for phosphothreonine site as shown in Table 1.

Casein kinase II phosphorylation sites were relatively more abundant than protein kinase $\mathrm{C}$ phosphorylation sites (amino acid residues range 51-54, 56-59, 209-212, 309-312, 38-341, 397-400, 417-420, and 640-643). N-myristoylation sites were abundant more than other functional sites in HDAC10. N-myristoylation sites are involved in the amino acids residues ranging from 88 to 93,120 to 125,136 to 141,217 to 222,219 to 224,232 to 237,273 to 278,294 to 299,384 to 389,469 
Table 1: Post-translational modifications (phosphorylation sites) of HDAC10

\begin{tabular}{|c|c|c|c|c|c|}
\hline \multirow[t]{2}{*}{ S.no } & \multirow[t]{2}{*}{ Site } & \multirow[t]{2}{*}{ Definition } & \multirow[t]{2}{*}{ Region } & \multicolumn{2}{|l|}{ Modified residue } \\
\hline & & & & Serine (S) & Threonine (T) \\
\hline 1. & PS00005 & $\begin{array}{l}\text { PKC_PHOSPHO_SITE, Protein kinase } \\
\text { C phosphorylation site }\end{array}$ & $51-53 ; 345-347 ; 409-411$ & $51 ; 345 ; 409$ & \\
\hline 2. & PS00006 & $\begin{array}{l}\text { CK2_PHOSPHO_SITE, casein kinase } \\
\text { II phosphorylation site }\end{array}$ & $\begin{array}{l}51-54 ; 56-59 ; 209-212 ; \\
309-312 ; 338-341 ; 397-400 \\
417-420 ; 640-643\end{array}$ & $51 ; 56 ; 209 ; 309 ; 338 ; 397 ; 640$ & 417 \\
\hline
\end{tabular}

HDAC10: Histone deacetylase 10

to 474,473 to 478,498 to 503,553 to 558 , and 599 to 604 . It is found that hydrophobic and steric interactions act in concert to anchor to cell membranes and proteins that are either myristoylated or farnesylated and myristoylation promotes the reversible membrane proteinprotein interactions (PPIs) [40] (Table 2). These results highlight $\mathrm{N}$-myristoylation, and Casein kinase II acts as a potential regulator of HDAC10 PTM and also shows that regulation of HDAC10 function may controlled in part by post-translational phosphorylation.

Protein domain boundaries and the knowledge of its architecture are an important aspect for understanding the protein function. Detection of protein domain and architecture is a valuable tool in the areas of protein science, protein structure prediction, etc., [41]. These setups allowed us to study the behavior of residues and its role in PTMs and motif functionality. PTM studies provide an extensible clue about protein's regulation process [42]. These features include the nature of signal transduction, protein stability, etc. [43]. The mechanism of transcriptional regulation of HDAC10 with respect to drug binding with phytocompounds remains poorly understood. Therefore, multiple strategies, including motif and domain, were determined to recognize binding sites to further study the docking and its dynamics. Thus, we hypothesize that a more reliable mechanism is highly desirable to determine the binding sites, binding mode, and its role in various cancers.

\section{Binding site determination}

Eight important binding pockets were determined as catalytic sites of the HDAC-10 receptor using metaPocket server. After clustering, the top three sites from the base methods, namely PASS11 (PAS), LigsiteCS (LCS), Q SiteFinder (QSF), GHECOM (GHE), POCASA (PCS), Fpocket (FPK), SURFNET (SFN) and ConCavity (CON), were the base methods incorporated to predict all the top eight clusters. MetaPocket running report is shown in Table 3. The first MetaPocket site consists of five pocket sites with total Z score of 19.45 from SFN-1, "LCS-1," "FPK-1," "CON-2," and "GHE-2." The second MetaPocket site consists of 6 pocket sites with a total Z score of 17.42 from GHE-1, "FPK-2," "LCS-2," "SFN2," "PAS-2," and "CON-1," and the third MetaPocket site consists of 1 pocket site with a total Z score of 2.51 from SFN-3. Eight header binding sites with potential binding site residues of the receptor are shown in Table 4. The identification of binding sites is an important step in structure-based drug design, as this helps to understand the functional sites and its mechanism of action of the protein [44]. These identified active sites were helpful to evaluate further the binding mode of docked complexes. Thus, we focused on the binding mode of the residues and evaluated its affinity to the HDAC10 receptor during the selected druginteraction studies through docking.

\section{HDAC10 and phytocompound binding mode analysis}

To analyze the reliability of interaction mode between HDAC10 and the compounds, namely periplogenin 3,3'-diindolylmethane and epigallocatechin, the binding energy score values were determined. Molecular docking is the standard method used to determine the binding mode of molecules with the receptors; docking was used $[45,46]$ in many studies. In our study, holacurtine showed a lesser energy value of -300.21 compared to other three compounds. The predicted interacting residues obtained by drug interaction studies were matched with the predicted binding pocket amino acids of MetaPocket server.
Table 2: Post-translational modifications (myristoylation sites) of HDAC10

\begin{tabular}{lll}
\hline S. No & Region & Residues \\
\hline 1. & $88-93$ & GQfdAI \\
2. & $120-125$ & GAvqNG \\
3. & $136-141$ & GQraAA \\
4. & $217-222$ & GQglGF \\
5. & $219-224$ & GLgfTV \\
6. & $232-237$ & GMgnAD \\
7. & $273-278$ & GQmqAT \\
8. & $294-299$ & GGrvCA \\
9. & $384-389$ & GGpvCK \\
10. & $469-474$ & GMldGQ \\
11. & $473-478$ & GQvnSG \\
12. & $498-503$ & GLshGA \\
13. & $553-558$ & GGflSC \\
14. & $599-604$ & GLagGR \\
\hline
\end{tabular}

HDAC10: Histone deacetylase 10

Table 3: Predicted ligand binding clusters site of receptor, HDAC10, using MetaPocket server

\begin{tabular}{|c|c|c|c|}
\hline Clusters & Z score & $\begin{array}{l}\text { Number of } \\
\text { pocket sites }\end{array}$ & Methods \\
\hline 1. & 19.45 & 5 & $\begin{array}{l}\text { “SFN-1," “LCS-1,” “FPK-1,” } \\
\text { “CON-2,” “GHE-2" }\end{array}$ \\
\hline 2. & 17.42 & 6 & $\begin{array}{l}\text { "GHE-1," “FPK-2," “LCS-2," } \\
\text { "SFN-2," "PAS-2," “CON-1" }\end{array}$ \\
\hline 3. & 2.51 & 1 & "SFN-3" \\
\hline 4. & 1.78 & 1 & "PAS-1" \\
\hline 5. & 0.99 & 1 & "LCS-3" \\
\hline 6. & 0.47 & 1 & "PAS-3" \\
\hline 7. & 0.44 & 1 & "GHE-3" \\
\hline 8. & -0.69 & 2 & "FPK-3," “CON-3" \\
\hline
\end{tabular}

HDAC10: Histone deacetylase 10

Holacurtine formed hydrogen bonds with Leu362, Ala363, Gln364, and Arg658 and steric interactions with Ser358, His361, Leu362, Ala363, Gln364, Val651, and Arg658. Holacurtine is only involving in the formation of hydrogen bond and hydrophobic bonds. Holacurtine does not form any hydrophobic bonds. In addition, inter-residue hydrogen bond interaction (Arg658-Glu655, Arg658-Met654, Arg658-Cys670, Glu652-Glu655, Glu655-Val651, and His361-Leu362) are involved in anchoring the holacurtine to the receptor HDAC10 (Fig. 1). Periplogenin forms hydrogen bond with Gln659, steric interactions with Arg658, Glu655, and Glu674, and hydrophobic interactions with His361. Arg658Cys670, Arg658-Glu655, Arg658-Leu668, Gln659-Glu655, and Glu674Trp676 forms inter-residue interactions with periplogenin as shown in Fig. 2. 3,3'-Diindolylmethane forms hydrogen bond interaction with Glu655, steric interactions with Arg349, Thr671, Arg658, and Glu655, and one hydrophobic bond with Cys670. Glu655-Val651, Arg349His246, Arg658-Cys670, Arg349-Phe359, Arg658-Glu655, Arg658Met654, and Cys670-His246 form inter-residue interactions with 3,3'-Diindolylmethane (Fig. 3). Epigallocatechin forms hydrogen bonds with Arg349, Arg658, Glu655, Arg349, and Gly567, steric interactions 
Table 4: Predicted ligand binding site residues in the receptor, HDAC10 using MetaPocket server

\begin{tabular}{|c|c|}
\hline Binding site & Residues \\
\hline Header binding site 1 & 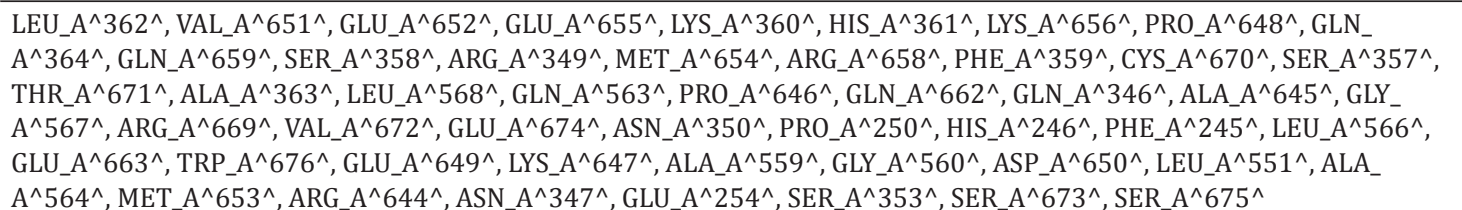 \\
\hline Header binding site 2 & 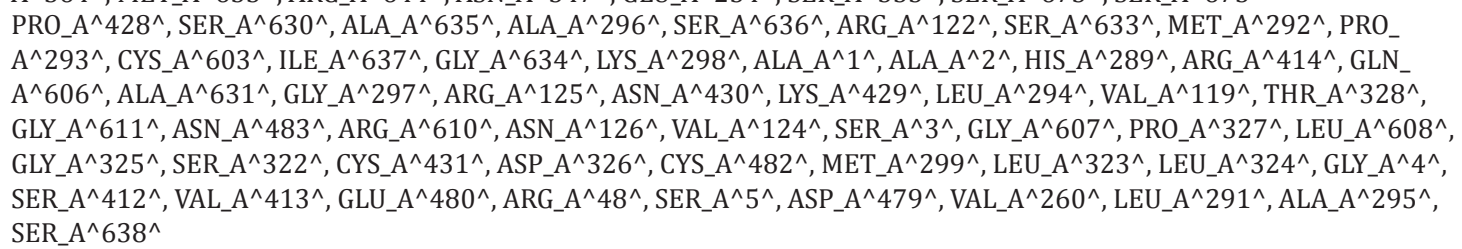 \\
\hline Header binding site3 & ILE_A^4 $437^{\wedge}$, VAL_A^ $4445^{\wedge}, \mathrm{LEU}_{-} \mathrm{A}^{\wedge} 462^{\wedge}, \mathrm{ARG} \mathrm{A}^{\wedge} 463^{\wedge}, \mathrm{SER} \_A^{\wedge} 464^{\wedge}, \mathrm{LEU} \_\mathrm{A}^{\wedge} 465^{\wedge}, \mathrm{GLY}_{-} \mathrm{A}^{\wedge} 466^{\wedge}$ \\
\hline Header binding site 4 & ASP_A^${ }^{\wedge} 22^{\wedge}, T_{Y R} A^{\wedge} 92^{\wedge}, A S P \_A^{\wedge} 94^{\wedge}$ \\
\hline Header binding site 6 & GLU_A^540^, ARG_A^ $552^{\wedge}$, MET_A $^{\wedge} 653^{\wedge}$ \\
\hline Header binding site 7 & 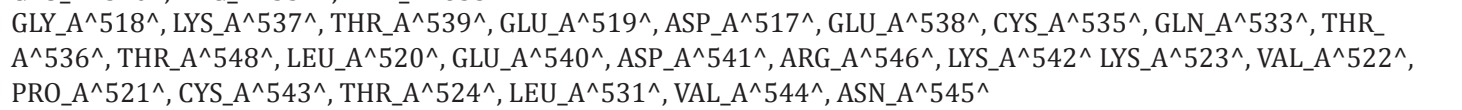 \\
\hline Header binding site 8 & 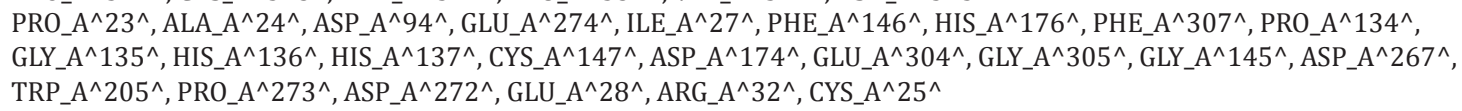 \\
\hline
\end{tabular}

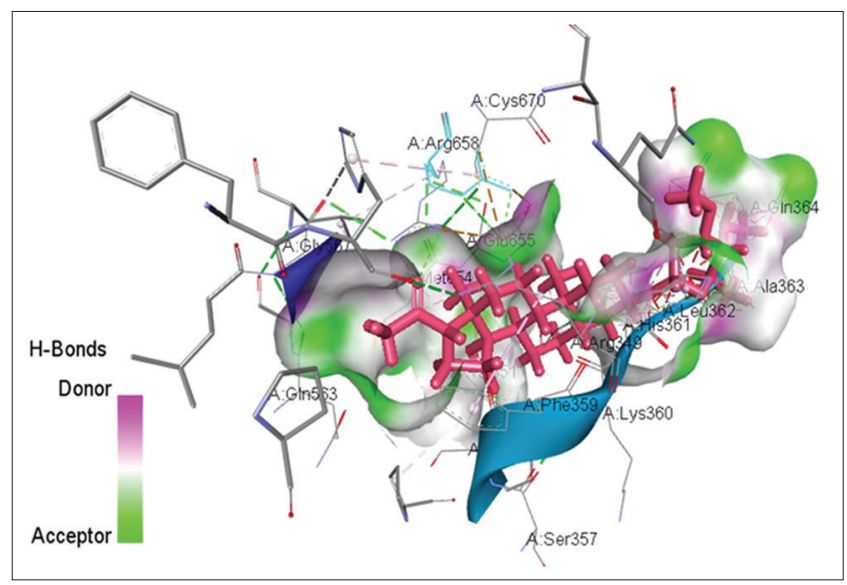

Fig. 1: Docking of holacurtine with histone deacetylase10 receptor showing interactions

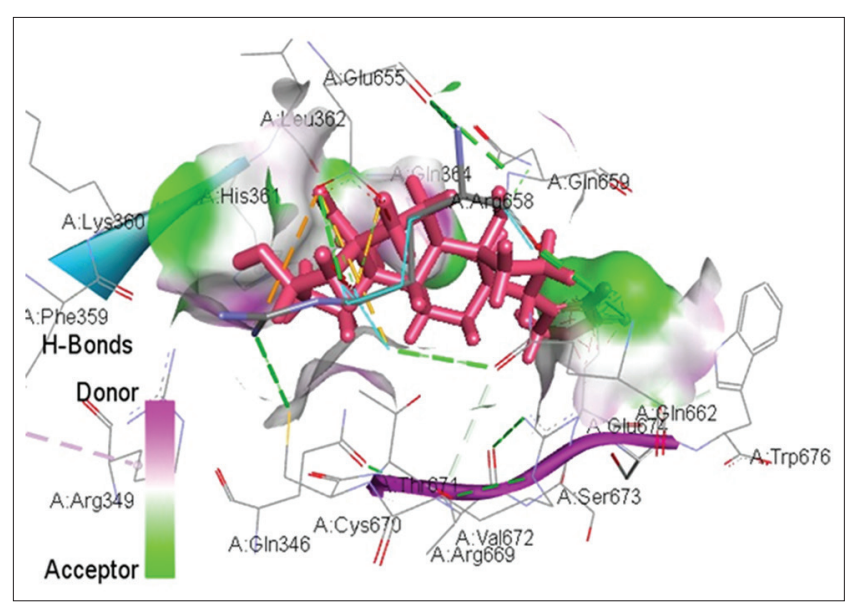

Fig. 2: Docking of periplogenin with histone deacetylase10 receptor showing interactions

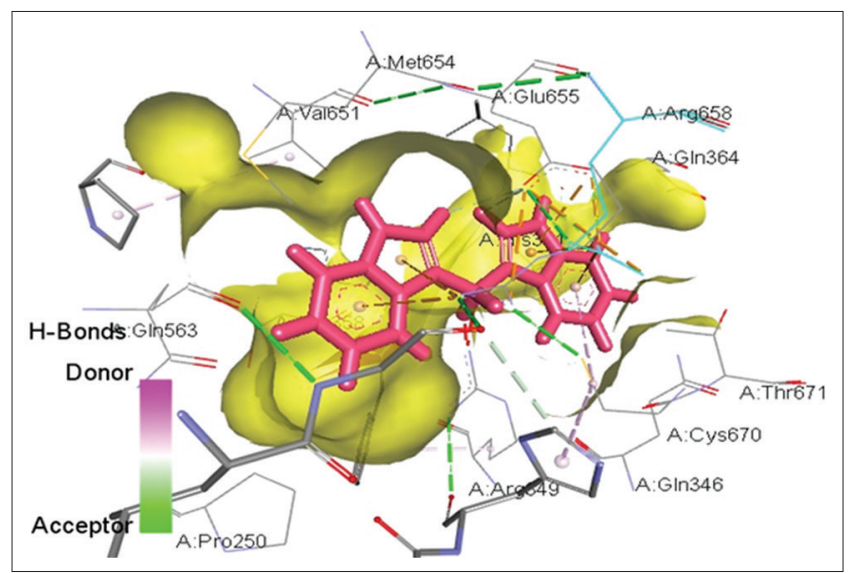

Fig. 3: Docking of 3,3'-diindolylmethane with histone deacetylase 10 receptor showing interactions

with Lys360 and Arg658, and hydrophobic interactions with His361. Arg349-His246, Arg349-Phe359, Arg658-Cys670, Arg658-Glu655, Arg658-Met654, Arg658-Lue568, Gly567-Gln563, and His361-Leu362 form inter-residue interactions with Epigallocatechin (Fig. 4).

Arg658 was found to be one of the critical amino acid residues which occurs in all the four molecules which form hydrogen bond and steric interactions in holacurtine and epigallocatechin and steric interactions in all the four compounds. Periplogenin, 3,3'-diindolylmethane, and epigallocatechin showed similar degree of hydrophobic residues (one residue each) that take part in hydrophobic interaction. Periplogenin and epigallocatechin have a consensus hydrophobic interaction residue His361; also the same residue His361 forms a steric interaction with holacurtine (Table 5).

Hydrophobic residues are critical determinants of ion selectivity. With more the hydrophobic residues, more will be the affinity and the overall effect will be greater for alkyl substituents. Studies suggest that ion selectivity mainly involves in cysteine residues [47]. Intermolecular binding shows the measure of binding stability between a protein and its binding components [48]. The 
predicted residue contacts information that will be able to predict interacting helical pairs and helix-helix interactions and predict residue contacts. Inter-residue atomic interactions including hydrogen bonds, hydrophobic interactions, salt bridges, and electrostatic (steric) interactions were computed between pairs of amino acid residues within the HDAC10 receptor. Since amino acid interactions are important determinants of protein foldfunction-evolution relationships [49], folding initiation sites contain not only accepted "hydrophobic" amino acids but also larger charged side chains [50].

Conservation of inter-residue interactions will be helpful to predict the folding rate of domain and repeats in proteins [51]. It has been found that, during the process of protein folding, the amino acid residues along the polypeptide chain interact with each other to form the stable native structure. The knowledge about inter-residue interactions in protein structures helped us to understand the mechanism of protein folding and stability [52]. From the above results of interaction energy and residues forming $\mathrm{H}$-bonds, hydrophobic interactions, and inter-residue interaction, four compounds were ranked in the order with respect to the predicted effectiveness of binding such as Holacurtine $>$ Periplogenin $>3$,3'-diindolylmethane $>$ epigallocatechin. Subsequently, 'Lipinski's rule of five' was performed to check the oral bioavailability of the molecules. Those molecules which do not satisfy one or more of Lipinski's rules were predicted to have issues with oral bioavailability. Molinspiration calculated all important molecular properties such as LogP, polar surface area, number of hydrogen bond donors and acceptors as presented in Table 6. TPSA (topological polar

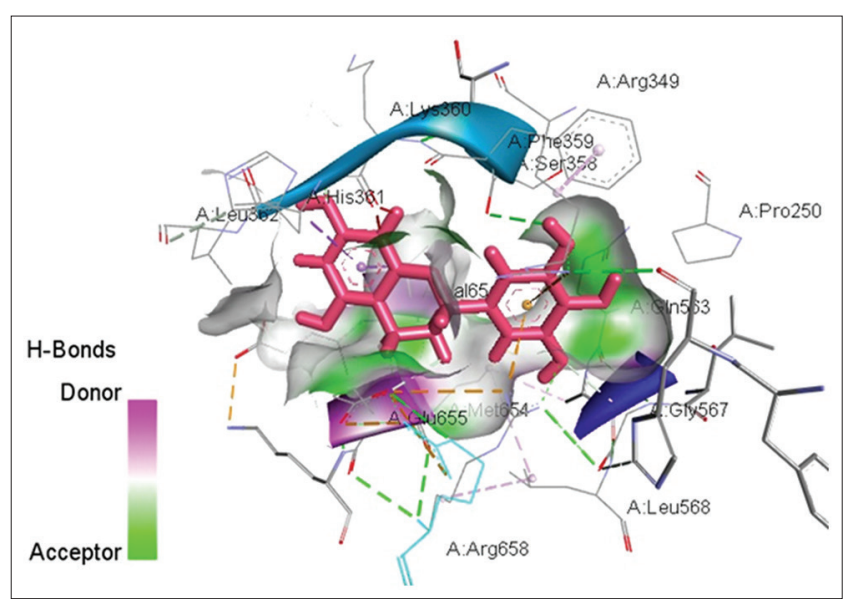

Fig. 4: Docking of epigallocatechin with histone deacetylase10 receptor showing interactions surface area) or PSA (polar surface area) is the term to describe the surface sum of all polar atoms, primarily oxygen and nitrogen, and attached hydrogens, generally PSA is used to calculate the percentage of absorption [53]. PSA is also considered as a suitable descriptor which determines H-bonding potential of drugs. For the molecules to penetrate the blood brain barrier, PSA value of less than 90 angstroms is preferred [54]. Taken together, our results demonstrated that binding site residues of HDAC10 may have undergone specific types of changes to develop various interactions that influence ligand anchorage and thus receptor-binding affinity of HDAC10. This study emphasizes that docking is one of the valuable methods to determine the receptor-drug interactions and to assess the affinity toward the receptor structures (Table 6).

Phytocompoundsare known to possess anti-inflammatory and antitumor activities which are involved in tumor growth and metastasis $[55,56]$ and also act as potential antibacterial components [57]. Although there exist various evidences and links between the phytocompounds and its interaction between HDACs [58-60], identification of binding sites and their binding mode is an important factor for each class of HDACs. It is mandatory to evaluate the specificity, drug-like characteristics, and pharmacokinetic profile of phytocompounds [61]. Major goal of structural biology deals with the formation of protein-ligand complexes. It has been demonstrated that an alkaloid, namely VI, was screened against various cancer cell lines and showed significant anticancer activity with $\mathrm{IC}_{50}$ value in the range of $0.89-1.40 \mu \mathrm{M}$ [62]. The role of receptor-ligand interaction helps to study the conceptual biology of protein function through docking analysis. Perceptive protein-ligand interactions will, therefore, be very important in drug discovery research. In this scenario, docking aids in identifying the transformation of one molecule to which other molecules can fit in devoid of any steric changes. This molecular docking study revealed the critical residues that take part in the HDAC10 binding mechanism. Our investigation revealed the overall accuracy and coverage values for drug binding mechanism of selected phytocompounds against HDAC10.

\section{CONCLUSION}

Our study demonstrated for the first time that, using the computational analysis, we could select four phytocompounds from the list of 450 phytocompounds. The selected compounds showed excellent binding interactions specifically for HDAC10. By employing docking and scoring analyses, we studied the binding mode of these four molecules and found that holacurtine, periplogenin, 3,3'-diindolylmethane, and epigallocatechin could potentially interact with HDAC10 in that order. These results provided valuable information about the essential structural features of effective HDAC10 inhibitors. These molecules

Table 5: Docking of the four compounds based on the docking score and interaction of residues

\begin{tabular}{|c|c|c|c|c|c|c|}
\hline S. No & $\begin{array}{l}\text { Compound name } \\
\text { (PubChem CID) }\end{array}$ & $\begin{array}{l}\text { Hydrogen bonds } \\
\text { interaction }\end{array}$ & Steric interaction & $\begin{array}{l}\text { Hydrophobic } \\
\text { interactions }\end{array}$ & $\begin{array}{l}\text { Docking } \\
\text { score }\end{array}$ & $\begin{array}{l}\text { ACE } \\
\text { value }\end{array}$ \\
\hline 1. & Holacurtine (10390928) & $\begin{array}{l}\text { Leu362, Ala363, Gln364, } \\
\text { Arg658, }\end{array}$ & $\begin{array}{l}\text { Ser358, His361, Leu362, Ala363, } \\
\text { Gln364, Val651, Arg658 }\end{array}$ & - & 6560 & -300.21 \\
\hline 2. & Periplogenin (10574) & Gln659 & Arg 658, Glu655, Glu674 & His361 & 4944 & -16.72 \\
\hline 3. & 3,3'-diindolylmethane (3071) & Glu655 & Arg349, Thr671, Arg658, Glu655 & Cys670 & 4676 & -102.21 \\
\hline 4. & Epigallocatechin (72277) & $\begin{array}{l}\text { Arg349, Arg658, Glu655, } \\
\text { Arg349, Gly567 }\end{array}$ & Lys 360 , Arg658 & His361 & 4220 & -136.75 \\
\hline
\end{tabular}

ACE: Atomic contact energy

Table 6: Drug likeness score for the four phytocompounds

\begin{tabular}{|c|c|c|c|c|c|c|c|c|c|}
\hline Molecule (PubChem compound id) & miLogP & TPSA & MW & $\mathrm{N}$ atoms & nON & nOHNH & nrotb & $\mathrm{N}$ violations & Volume \\
\hline Holacurtine (10390928) & 4.04 & 77.03 & 491.71 & 35 & 6 & 2 & 5 & 0 & 493.13 \\
\hline Periplogenin (10574) & 1.53 & 86.99 & 390.52 & 28 & 5 & 3 & 1 & 0 & 372.33 \\
\hline 3,3'-Diindolylmethane (3071) & 4.20 & 31.58 & 246.31 & 19 & 2 & 2 & 2 & 0 & 230 \\
\hline Epigallocatechin (72277) & 1.08 & 130.60 & 306.27 & 22 & 7 & 6 & 1 & 0 & 252.16 \\
\hline
\end{tabular}


could be validated to check its $\mathrm{ADME}, \mathrm{IC}_{50}$ value, and anti-inflammatory responses using both in vitro and in vivo methods. The computational observations of PTM together with the binding mode information have given information in the field of SNP and mutation studies as well as paved the way for new research approach for efficiently selecting critical regions and sites including non-coding residues for drug binding domains which may have great scope in cancer research. Furthermore, the PTM studies could form a starting point to further understand its PPI with TXNIP protein and regulation mechanisms which is still not yet determined. The docking and dynamics score studies demonstrated the selection procedure of phytocompounds toward the receptor, and these methodologies might be helpful for developing new drugs for HDAC10 protein driven cancers.

\section{ACKNOWLEDGMENTS}

The authors are grateful to JNIAS, Hyderabad, Telangana, for the facilities provided.

\section{AUTHOR'S CONTRIBUTION}

S.M. conceived the idea, participated in the study design, performed data collection and analysis, and drafted the manuscript under K.J.'s supervision. K.J. designed and supervised the study, coordinated the data collection, analysis, and its interpretation, helped in drafting the manuscript, and provided the critical revisions. A.V.N.S. coordinated the study and gave useful suggestions. All authors read and gave final approval for publication.

\section{CONFLICTS OF INTEREST}

The authors declared that there are no conflicts of interest.

\section{REFERENCES}

1. Grunstein M. Histone acetylation in chromatin structure and transcription. Nature 1997;389:349-52.

2. Koprinarova M, Schnekenburger M, Diederich M. Role of histone acetylation in cell cycle regulation. Curr Top Med Chem 2016;16:732-44.

3. Wright DE, Wang CY, Kao CF. Histone ubiquitylation and chromatin dynamics. Front Biosci (Landmark Ed) 2012;17:1051-78.

4. Kuo MH, Allis CD. Roles of histone acetyltransferases and deacetylases in gene regulation. Bioessays 1998;20:615-26.

5. Delcuve GP, Khan DH, Davie JR. Roles of histone deacetylases in epigenetic regulation: Emerging paradigms from studies with inhibitors. Clin Epigenetics 2012;4:5.

6. Hagelkruys A, Sawicka A, Rennmayr M, Seiser C. The biology of HDAC in cancer: The nuclear and epigenetic components. Handb Exp Pharmacol 2011;206:13-37.

7. Warrell RP Jr., He LZ, Richon V, Calleja E, Pandolfi PP. Therapeutic targeting of transcription in acute promyelocytic leukemia by use of an inhibitor of histone deacetylase. J Natl Cancer Inst 1998;90:1621-5.

8. Ropero S, Esteller M. The role of histone deacetylases (HDACs) in human cancer. Mol Oncol 2007;1:19-25.

9. Pang M, Zhuang S. Histone deacetylase: A potential therapeutic target for fibrotic disorders. J Pharmacol Exp Ther 2010;335:266-72.

10. Falkenberg KJ, Johnstone RW. Histone deacetylases and their inhibitors in cancer, neurological diseases and immune disorders. Nat Rev Drug Discov 2014;13:673-91.

11. Kim HJ, Bae SC. Histone deacetylase inhibitors: Molecular mechanisms of action and clinical trials as anti-cancer drugs. Am J Transl Res 2011;3:166-79.

12. Dokmanovic M, Marks PA. Prospects: Histone deacetylase inhibitors. J Cell Biochem 2005;96:293-304.

13. Ren J, Zhang J, Cai H, Li Y, Zhang Y, Zhang X, et al. HDAC as a therapeutic target for treatment of endometrial cancers. Curr Pharm Des 2014:20:1847-56.

14. Bolden JE, Peart MJ, Johnstone RW. Anticancer activities of histone deacetylase inhibitors. Nat Rev Drug Discov 2006;5:769-84.

15. Tambunan US, Wulandari EK. Identification of a better homo sapiens class II HDAC inhibitor through binding energy calculations and descriptor analysis. BMC Bioinformatics 2010;11 Suppl 7:S16.

16. Marks PA, Richon VM, Rifkind RA. Histone deacetylase inhibitors: Inducers of differentiation or apoptosis of transformed cells. J Natl Cancer Inst 2000;92:1210-6.
17. Tong JJ, Liu J, Bertos NR, Yang XJ. Identification of HDAC10, a novel class II human histone deacetylase containing a leucine-rich domain. Nucleic Acids Res 2002;30:1114-23.

18. Yang Y, Huang Y, Wang Z, Wang HT, Duan B, Ye D, et al. HDAC10 promotes lung cancer proliferation via AKT phosphorylation. Oncotarget 2016;7:59388-401.

19. Song C, Zhu S, Wu C, Kang J. Histone deacetylase (HDAC) 10 suppresses cervical cancer metastasis through inhibition of matrix metalloproteinase (MMP) 2 and 9 expression. J Biol Chem 2013;288:28021-33

20. Islam MM, Banerjee T, Packard CZ, Kotian S, Selvendiran K, Cohn DE, et al. HDAC10 as a potential therapeutic target in ovarian cancer. Gynecol Oncol 2017;144:613-20.

21. Guardiola AR, Yao TP. Molecular cloning and characterization of a novel histone deacetylase HDAC10. J Biol Chem 2002;277:3350-6.

22. Lee JH, Jeong EG, Choi MC, Kim SH, Park JH, Song SH, et al. Inhibition of histone deacetylase 10 induces thioredoxin-interacting protein and causes accumulation of reactive oxygen species in SNU620 human gastric cancer cells. Mol Cells 2010;30:107-12.

23. Jamil K, Mustafa SM. Thioredoxin system: A model for determining novel lead molecules for breast cancer chemotherapy. Avicenna J Med Biotechnol 2012;4:121-30.

24. Osada H, Tatematsu Y, Saito H, Yatabe Y, Mitsudomi T, Takahashi T, et al. Reduced expression of class II histone deacetylase genes is associated with poor prognosis in lung cancer patients. Int J Cancer 2004; 112:26-32.

25. Oehme I, Lodrini M, Brady NR, Witt O. Histone deacetylase 10-promoted autophagy as a druggable point of interference to improve the treatment response of advanced neuroblastomas. Autophagy 2013;9:2163-5.

26. Wang JC, Kafeel MI, Avezbakiyev B, Chen C, Sun Y, Rathnasabapathy C, et al. Histone deacetylase in chronic lymphocytic leukemia. Oncology 2011;81:325-9.

27. Coiffier B, Federico M, Caballero D, Dearden C, Morschhauser F, Jäger $\mathrm{U}$, et al. Therapeutic options in relapsed or refractory peripheral T-cell lymphoma. Cancer Treat Rev 2014;40:1080-8.

28. Raedler LA. Farydak (Panobinostat): First HDAC inhibitor approved for patients with relapsed multiple myeloma. Am Health Drug Benefits 2016;9:84-7.

29. Magrane M, UniProt Consortium. UniProt knowledgebase: A hub of integrated protein data. Database (Oxford) 2011;2011:bar009.

30. Hai Y, Shinsky SA, Porter NJ, Christianson DW. Histone deacetylase 10 structure and molecular function as a polyamine deacetylase. Nat Commun 2017;8:15368.

31. Zhang Z, Li Y, Lin B, Schroeder M, Huang B. Identification of cavities on protein surface using multiple computational approaches for drug binding site prediction. Bioinformatics 2011;27:2083-8.

32. Wang Y, Xiao J, Suzek TO, Zhang J, Wang J, Bryant SH, et al. PubChem: A public information system for analyzing bioactivities of small molecules. Nucleic Acids Res 2009;37:W623-33.

33. O'Boyle NM, Banck M, James CA, Morley C, Vandermeersch T, Hutchison GR, et al. Open babel: An open chemical toolbox. J Cheminform 2011;3:33.

34. Duhovny D, Nussinov R, Wolfson HJ. Efficient Unbound Docking of Rigid Molecules. Berlin Heidelberg: Springer; 2002. p. 185-200.

35. Schneidman-Duhovny D, Inbar Y, Nussinov R, Wolfson HJ. PatchDock and symmDock: Servers for rigid and symmetric docking. Nucleic Acids Res 2005;33:W363-7.

36. Voruganti HK, Dasgupta B. A novel volumetric criterion for optimal shape matching of surfaces for protein-protein docking. J Comput Des Eng 2018;5:180-90.

37. Benyamini H, Shulman-Peleg A, Wolfson HJ, Belgorodsky B, Fadeev L, Gozin $\mathrm{M}$, et al. Interaction of $\mathrm{c}(60)$-fullerene and carboxyfullerene with proteins: Docking and binding site alignment. Bioconjug Chem 2006; 17:378-86.

38. Lipinski CA, Lombardo F, Dominy BW, Feeney PJ. Experimental and computational approaches to estimate solubility and permeability in drug discovery and development settings. Adv Drug Deliv Rev 2001;46:3-26.

39. Kishimoto A, Nishiyama K, Nakanishi H, Uratsuji Y, Nomura H, Takeyama Y, et al. Studies on the phosphorylation of myelin basic protein by protein kinase $\mathrm{C}$ and adenosine 3':5'-monophosphatedependent protein kinase. J Biol Chem 1985;260:12492-9.

40. Murray D, Ben-Tal N, Honig B, McLaughlin S. Electrostatic interaction of myristoylated proteins with membranes: Simple physics, complicated biology. Structure 1997;5:985-9.

41. Kong L, Ranganathan S. Delineation of modular proteins: Domain 
boundary prediction from sequence information. Brief Bioinform 2004;5:179-92.

42. Dewhurst HM, Torres MP. Systematic analysis of non-structural protein features for the prediction of PTM function potential by artificial neural networks. PLoS One 2017; 12:e0172572.

43. Audagnotto M, Dal Peraro M. Protein post-translational modifications: In silico prediction tools and molecular modeling. Comput Struct Biotechnol J 2017;15:307-19.

44. Chakrabarti S, Lanczycki CJ. Analysis and prediction of functionally important sites in proteins. Protein Sci 2007;16:4-13.

45. Begum A, Begum S, Kvsrg P, Bharathi K. In silico studies on functionalized azaglycine derivatives containing 2, 4-thiazolidinedione scaffold on multiple targets. Int J Pharm Pharm Sci 2017;9:62-75.

46. Singh AN, Baruah MM, Sharma N. Structure based docking studies towards exploring potential anti-androgen activity of selected phytochemicals against prostate cancer. Sci Rep 2017;7:1955.

47. Hansen SB, Wang HL, Taylor P, Sine SM. An ion selectivity filter in the extracellular domain of cys-loop receptors reveals determinants for ion conductance. J Biol Chem 2008;283:36066-70.

48. Stetz G, Verkhivker GM. Functional role and hierarchy of the intermolecular interactions in binding of protein kinase clients to the Hsp90-Cdc37 chaperone: Structure-based network modeling of allosteric regulation. J Chem Inf Model 2018;58:405-21.

49. Chou KC, Zhang CT. Predicting protein folding types by distance functions that make allowances for amino acid interactions. J Biol Chem 1994;269:22014-20.

50. Dyson HJ, Wright PE, Scheraga HA. The role of hydrophobic interactions in initiation and propagation of protein folding. Proc Natl Acad Sci U S A 2006;103:13057-61.

51. Mary RD, Saravanan MK, Selvaraj S. Conservation of inter-residue interactions and prediction of folding rates of domain repeats. J Biomol Struct Dyn 2015;33:534-51.

52. Gromiha MM, Selvaraj S. Inter-residue interactions in protein folding and stability. Prog Biophys Mol Biol 2004;86:235-77.

53. Schaftenaar G, de Vlieg J. Quantum mechanical polar surface area. J Comput Aided Mol Des 2012;26:311-8.

54. Bartzatt R. Lomustine analogous drug structures for intervention of brain and spinal cord tumors: The benefit of in silico substructure search and analysis. Chemother Res Pract 2013;2013:360624.

55. Johnson IT. Phytochemicals and cancer. Proc Nutr Soc 2007;66:207-15.

56. Yin SY, Yang NS, Lin TJ. Phytochemicals approach for developing cancer immunotherapeutics. front pharmacol. Front Med 2017;8:386.

57. zMalar CG, Chellaram C. Studies on phytochemical screening, antioxidant activity and anti-bacterial activity of salacia oblonga stem extract. Int J Pharm Pharm Sci 2016;8:32-6

58. Upadhyay S, Dixit M. Role of polyphenols and other phytochemicals on molecular signaling. Oxid Med Cell Longev 2015;2015:504253.

59. Thakur VS, Deb G, Babcook MA, Gupta S. Plant phytochemicals as epigenetic modulators: Role in cancer chemoprevention. AAPS J 2014;16:151-63.

60. Farrand L, Oh S-W, Song YS, Tsang BK. Phytochemicals: A multitargeted approach to gynecologic cancer therapy. Biomed Res Int 2014;2014:890141.

61. Losson H, Schnekenburger M, Dicato M, Diederich M. Natural compound histone deacetylase inhibitors (HDACi): Synergy with inflammatory signaling pathway modulators and clinical applications in cancer. Molecules 2016;21: pii: E1608.

62. Dhiman M, Khanna A, Manju S. A new phenanthroindolizidine alkaloid from Tylophora indica. Chem Pap 2013;67:245-8. 